\title{
Transition to chaos in a cross-corrugated channel at low Reynolds numbers
}

\author{
Zhu, Xiaowei; Walther, Jens Honore; Zhao, Dan; Haglind, Fredrik
}

Published in:

Physics of Fluids

Link to article, DOI:

$10.1063 / 1.5122305$

Publication date:

2019

Document Version

Publisher's PDF, also known as Version of record

Link back to DTU Orbit

Citation (APA):

Zhu, X., Walther, J. H., Zhao, D., \& Haglind, F. (2019). Transition to chaos in a cross-corrugated channel at low Reynolds numbers. Physics of Fluids, 31(11), [114107]. https://doi.org/10.1063/1.5122305

\section{General rights}

Copyright and moral rights for the publications made accessible in the public portal are retained by the authors and/or other copyright owners and it is a condition of accessing publications that users recognise and abide by the legal requirements associated with these rights.

- Users may download and print one copy of any publication from the public portal for the purpose of private study or research.

- You may not further distribute the material or use it for any profit-making activity or commercial gain

- You may freely distribute the URL identifying the publication in the public portal

If you believe that this document breaches copyright please contact us providing details, and we will remove access to the work immediately and investigate your claim 


\section{Transition to chaos in a cross-corrugated channel at low Reynolds numbers}

Cite as: Phys. Fluids 31, 114107 (2019); https://doi.org/10.1063/1.5122305

Submitted: 30 July 2019 . Accepted: 28 October 2019 . Published Online: 26 November 2019

Xiaowei Zhu (D), Jens Honore Walther, Dan Zhao (D), and Fredrik Haglind

COLLECTIONS

F This paper was selected as Featured
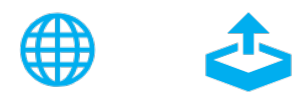

\section{ARTICLES YOU MAY BE INTERESTED IN}

Effect of soluble surfactant on the motion of a confined droplet in a square microchannel Physics of Fluids 31, 117104 (2019); https://doi.org/10.1063/1.5125949

Flow structures in transitional and turbulent boundary layers

Physics of Fluids 31, 111301 (2019); https://doi.org/10.1063/1.5121810

Numerical study of total temperature effect on hypersonic boundary layer transition Physics of Fluids 31, 114105 (2019); https://doi.org/10.1063/1.5125116

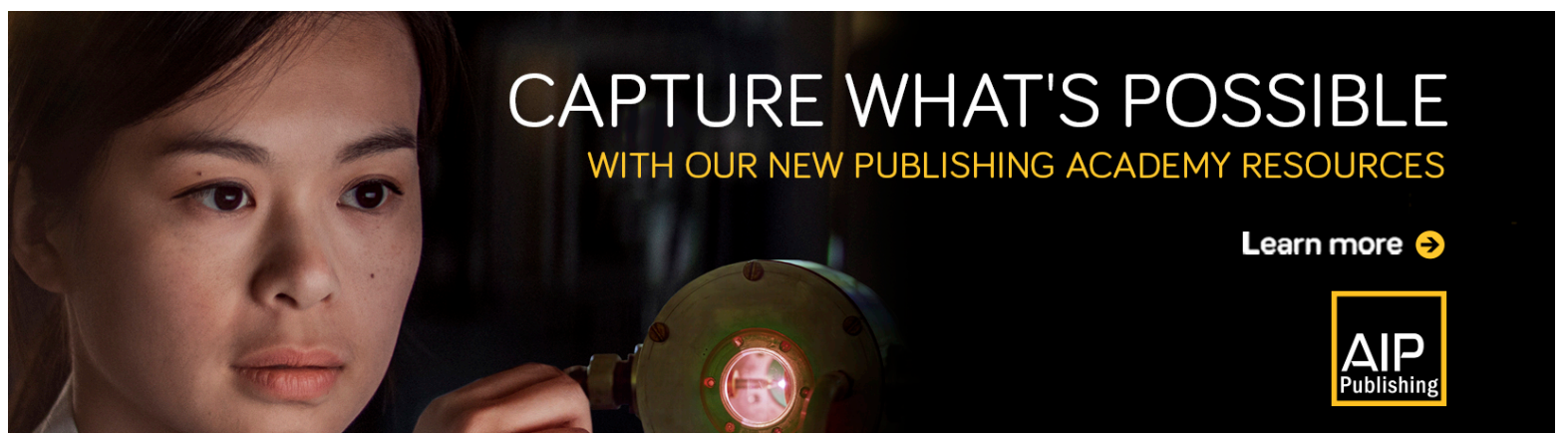




\title{
Transition to chaos in a cross-corrugated channel at low Reynolds numbers
}

\author{
Cite as: Phys. Fluids 31, 114107 (2019); doi: 10.1063/1.5122305 \\ Submitted: 30 July 2019 - Accepted: 28 October 2019 • \\ Published Online: 26 November 2019
}

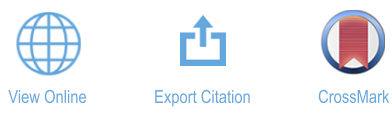

\author{
Xiaowei Zhu, ${ }^{1}$ (D) Jens Honore Walther, ${ }^{1,2}$ Dan Zhao, ${ }^{3}$ (D) and Fredrik Haglind \\ AFFILIATIONS \\ ${ }^{1}$ Department of Mechanical Engineering, Technical University of Denmark, Nils Koppels Allé, Building 403, \\ 2800 Kongens Lyngby, Denmark \\ ${ }^{2}$ Computational Science and Engineering Laboratory, ETH Zürich, Clausiusstrasse 33, CH-8092 Zürich, Switzerland \\ ${ }^{3}$ Department of Mechanical Engineering, University of Canterbury, Christchurch 8140, New Zealand
}

\begin{abstract}
The pressure-gradient driven flow in a three-dimensional cross-corrugated channel is investigated based on large eddy simulations. The channel geometry is highly tortuous so that the flow unsteadiness can be triggered at a moderate Reynolds number. The objective of this paper is to provide a better understanding of the laminar-to-chaotic transition in this type of channel. The transition from steady to chaos is found to occur at a low Reynolds number range between 77.4 and 113.1. The nonlinear dynamics is analyzed based on the power spectra of the velocity and reconstructed phase space. The route to chaos is identified, which favors the Ruelle-Takens-Newhouse scenario. Moreover, the transition from quasiperiodic mode to chaotic mode is accompanied with temporal intermittencies. The fluid dynamics is analyzed. It is found that the cross-corrugated geometry prompts a recirculating-and-rotating wake behind each contact corner. Each wake is enfolded by a pair of curved free shear layers. They are destabilized by the Kelvin-Helmholtz instability, leading to the periodic flow oscillation. Subsequently, the centrifugal instability sets in and promotes a type of primary vortex structures, forming streamwise "zig-zag" vortex streets. The competition between the adjacent vortex streets leads to a quasiperiodic flow. Temporal intermittencies emerge as the Reynolds number is increased. Finally, the periodicities in both the streamwise and spanwise directions are broken, and the flow becomes chaotic. When further increasing the Reynolds number to 343.1, Taylor-Görtler-like vortexes and necklace-like vortexes are formed in the channel.
\end{abstract}

Published under license by AIP Publishing. https://doi.org/10.1063/1.5122305

\section{INTRODUCTION}

This article brings into focus a typical flow channel, which is confined by two corrugated sheets that are attached in a crisscross fashion, hence, entitled a "cross-corrugated channel;" see Fig. 1. The cross-corrugated geometry can exert a strong disturbance on the internal flow to promote eddies, thereby enhancing the fluid mixing and the heat and mass transfer. Owing to this, the cross-corrugated geometry has been widely involved in many engineering designs, such as the plate heat exchanger, ${ }^{1}$ structured packing, ${ }^{2}$ microturbine recuperator, ${ }^{3}$ and static mixer.

Focke and Knibbe ${ }^{5}$ looked into the flow characteristics in crosscorrugated channels via experimental flow visualizations. The flow paths are sensitive to the intersection angle $\beta$ between the opposite plates. The stream traces either the furrow-wise direction at small $\beta\left(<45^{\circ}\right)$ or the streamwise direction when $\beta$ is large. Specifically, pairs of "ox-horn"-shaped vortexes were observed for $10<R e<200$, while the physical mechanism for the formation of the vortex pairs was not provided. The authors conjectured that there probably coexist other vortex structures in the channel which were not detected in the experiment, implying the complicity of the flow structure in such kinds of channels. Aside from the experiment, some computational fluid dynamics (CFD) simulations of cross-corrugated channel flows have been done in the past two decades. Most of them are mainly purposed on the prediction of global heat transfer or pressure drop (e.g., Tsai et al., ${ }^{6}$ Freund et al., ${ }^{7}$ Sarraf et al., ${ }^{8}$ and Chapaloglou et $\left.a l .{ }^{9}\right)$ rather than on a better understanding of the fluid behavior inside the channel. Blomerius et al. ${ }^{10}$ performed a numerical simulation of flow in cross-corrugated channels $\left(\beta=45^{\circ}\right)$ with $150<\operatorname{Re}$ $<2000$ and found that the self-sustained oscillation occurs as $R e$ is beyond a critical value of 240 and the flow becomes chaotic when $R e$ gets even higher. Lee and Lee ${ }^{11}$ performed large eddy simulations of the flow in cross-corrugated channels. They detected periodic oscillations of the flow at $R e=850$. The research mentioned above 
suggests that the transition to the unsteadiness of a cross-corrugated channel flow starts at a moderate Re usually in the order of $10^{2}$.

The transition to chaos in confined channels is of fundamental fluid dynamical interest. The flow transition in complex geometric channels is usually attained at a very low $R e$, and it is generally associated with the early onset of hydraulic instabilities caused by geometric modifications, such as surface grooves, transverse furrows, fins, ribs, blocks, and surface curvatures. Ghaddar et al. ${ }^{12}$ found that the first instability in a grooved channel is the KelvinHelmholtz $(\mathrm{K}-\mathrm{H})$ type. It destabilizes the free shear into the vortex sheet, leading to the self-excited oscillation closely resembling Tollmien-Schlichting modes. This principle generally holds in a large range of modified channels such as obstructed channels, ${ }^{1 .}$ channels with backward-facing steps, ${ }^{14}$ channels with small cylinder insertions, ${ }^{15}$ and converging-diverging channels. ${ }^{16}$ Roberts ${ }^{13}$ experimentally investigated the flow transition in an obstructed channel. Free shear layers are formed at the edge of the obstacles, which undergo a transition at $R e \sim 100$, leading to the periodic flow oscillation. It was then followed by a secondary transition at $R e \sim 160$, and the flow became chaotic. Robert and Mackley ${ }^{17}$ further examined the transition process in the obstructed channel based on the two-dimensional numerical simulation. They found that the flow experiences a periodic doubling sequence of transition to chaos. Guzmán and Amon ${ }^{16}$ performed direct numerical simulations of the flow in two-dimensional converging-diverging channels. By increasing the control parameter $R e$, the flow transition undergoes a sequence of steady, periodic, quasiperiodic-1, period with phaselocking, quasiperiodic-2, and chaos, which conforms with the transition route described by the Ruelle-Takens-Newhouse scenario. Sui et al. ${ }^{18}$ examined the flow transition in a three-dimensional wavy channel. The flow bifurcates from periodic state to quasiperiodic mode (QP) via the emergence of an incommensurate frequency at $R e=333$, and finally goes toward chaos at $R e=400$. The transition sequence also favors the Ruelle-Takens-Newhouse scenario. In contrast to the aforementioned ribbed channels, converging-diverging channels, and wavy channels whose internal flow transitions have been frequently investigated, the cross-corrugated channel has not received any attention in terms of the flow transition mechanisms. It is not clear how the flow transition takes place inside this kind of channel.

The transition to chaos in confined channels is crucial for engineering practice as well. Taking the heat exchanger as an example, the chaotic mixing in the flow passages can substantially enhance the heat and mass transfer coefficients compared to the laminar flow. On the other hand, the chaos can lead to undesired increase of momentum transport as measured by the friction or the pressure loss. Therefore, the overall thermal-hydraulic performances of a heat exchanger operating under laminar and turbulent conditions are completely different. It is of crucial importance to identify the critical transition point in order to ascertain whether the flow condition is laminar or turbulent. For instance, it is well-known that the critical Reynolds number for the pipe flow is around 2300. However, the critical transition Reynolds number in a cross-corrugated channel has not been investigated.

This study is purposed on the exploration of the basic fluid dynamics in a cross-corrugated channel with the focus centered on the laminar-to-chaotic transition regime. We use large eddy simulations to acquire the flow information in the cross-corrugated channel for $9.3 \leq R e \leq 343.1$. Within this $R e$ range, the flow transition in the considered channel takes place. The nonlinear dynamics and the fluid dynamics during the flow transition are analyzed in detail. The main goal is to identify and interpret the sequence of transition from steady to chaos in the considered cross-corrugated channel. The understanding of this transitional process will be beneficial for the modeling and design of a range of engineering products involving the cross-corrugated channel.

This paper is organized as follows: In Sec. II, numerical methods are presented. The flow transition from steady to chaos is analyzed and discussed in Sec. III. In Sec. IV, the route to chaos is profiled using the spectra map, the Poincaré section, and the bifurcation diagram. The conclusions are provided in Sec. V.

\section{NUMERICAL METHODS}

\section{A. Governing equations}

In the present study, the flow field in the cross-corrugated channel is simulated based on large eddy simulations. For the incompressible flow, the governing equations for mass and momentum conservations are

$$
\begin{gathered}
\frac{\partial \tilde{u}_{i}}{\partial x_{i}}=0 \\
\rho \frac{\partial \tilde{u}_{i}}{\partial t}+\rho \frac{\partial \tilde{u}_{i} \tilde{u}_{j}}{\partial x_{j}}=-\frac{\partial \tilde{p}}{\partial x_{i}}+\frac{\partial}{\partial x_{j}}\left[\mu\left(\frac{\partial \tilde{u}_{i}}{\partial x_{j}}+\frac{\partial \tilde{u}_{j}}{\partial x_{i}}\right)\right]-\frac{\partial \tau_{i j}}{\partial x_{j}} .
\end{gathered}
$$

The subgrid turbulent stress in the momentum equation is approximated by the Boussinesq hypothesis, with $\tau_{i j}-\frac{1}{3} \tau_{k k} \delta_{i j}=-2 \mu_{t} \tilde{S}_{i j}$. The "wall adapted local eddy-viscosity" (WALE) model ${ }^{19}$ is used to account for the subgrid eddy viscosity, which is given in the form of
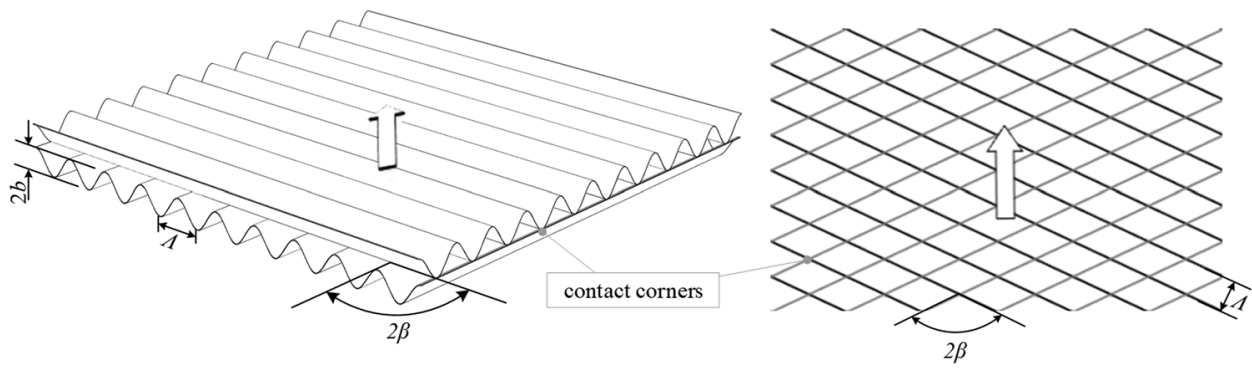

FIG. 1. A sketch of the cross-corrugated geometry with side view (left) and top view (right). Contact corners are the places at which the two opposite plates get attached. The main flow usually follows the direction indicated by the arrow. 


$$
\mu_{t}=\rho\left(C_{w} \Delta\right)^{2} \frac{\left(S_{i j}^{d} S_{i j}^{d}\right)^{3 / 2}}{\left(\tilde{S}_{i j} \tilde{S}_{i j}\right)^{5 / 2}+\left(S_{i j}^{d} S_{i j}^{d}\right)^{5 / 4}},
$$

where $C_{w}=0.325, \Delta=V^{1 / 3}$ ( $V$ denotes the cell volume), $\tilde{S}_{i j}$ $=\frac{1}{2}\left(\frac{\partial \tilde{u}_{i}}{\partial x_{j}}+\frac{\partial \tilde{u}_{j}}{\partial x_{i}}\right)$, and $S_{i j}^{d}=\frac{1}{2}\left[\left(\frac{\partial \tilde{u}_{i}}{\partial x_{j}}\right)^{2}+\left(\frac{\partial \tilde{u}_{j}}{\partial x_{i}}\right)^{2}\right]-\frac{1}{3} \delta_{i j}\left(\frac{\partial \tilde{u}_{k}}{\partial x_{k}}\right)^{2}$.

For the case of pure (laminar) shear, $S_{i j}^{d} S_{i j}^{d}=0$, and hence, $\mu_{t}$ $=0$ and the subgrid scale tensor is switched off. This leads to a natural decay of the subgrid eddy viscosity in the vicinity of the wall obeying the law of $\mu_{t} \sim\left(y^{+}\right)^{3}$. The eddy viscosity in free shear layers remote from walls can also be well represented by WALE, ${ }^{20}$ making it suitable for dealing with transition flows.

The governing equations are solved by using a finite-volume solver, ANSYS Fluent v18.2. The pressure-implicit with the splitting of operators (PISO) scheme is adopted for pressure-velocity coupling. The convective term is discretized by the central differencing scheme. The temporal discretization is based on a second-order backward differencing scheme. The time step is refined to ensure that the maximum Courant number of the flow field is smaller than unity.

\section{B. Computational domain and grids}

The geometry of the cross-corrugated channel can be characterized by three parameters: the corrugation amplitude $b$, the corrugation pitch $\Lambda$, and the intersection angle $\beta$, as marked in Fig. 1 . In the present study, the dimensions of three parameters are set to $b=1 \mathrm{~mm}, \Lambda=7 \mathrm{~mm}$, and $\beta=65^{\circ}$, respectively. This set of geometrical dimensions is widely adopted for the commercial plate heat exchanger design. The hydraulic diameter $d_{h}$ is calculated with $d_{h}$ $=4 V_{c} / A_{c}$, where $V_{c}$ and $A_{c}$ are the volume and the wet surface area of the channel, respectively. The Reynolds number is defined as $R e$ $=\rho U d_{h} / \mu$, where $\rho$ and $\mu$ are the fluid density and the viscosity, respectively; $\bar{U}$ is the characteristic velocity, equaling to the mean volumetric flow rate divided by the cross section area of the channel. In order to reduce the computational cost, only a repetitive cell of the channel is considered as the computational domain, as illustrated in Fig. 2(a). The contact corner between two plates is treated as a tiny cylinder with a superficial height of $0.01 d_{h}$. Hexahedral grids are formulated for the domain. Four numerical probes are placed in the domain to collect the transient flow information and are marked in Fig. 2(b).

In order to find a proper grid size, we tested three sets of grids, with grid sizes of 512125 (coarse), 1989757 (medium), and 4105950 (fine) cells, respectively. The test was performed at $R e=343.1$. The near wall grid was refined to ensure $y^{+}<1$. The mean flow properties at the middle [the C1-to-C2 line marked in Fig. 2(b)] of the domain were examined to find out a proper grid size. From Fig. 3, it is obvious to see that the mean velocity profiles, and the root-mean-square of velocity fluctuations, from the coarse grid deviate from the other two, whereas the medium grid shows a good resolution comparable to the fine grid. Then, it can be assumed that the medium grid size is ample for $R e \leq 343.1$. It is therefore adopted for all simulations throughout this paper.

\section{Boundary conditions}

The no-slip boundary condition is implemented to corrugated walls, and periodic boundary conditions are applied to both the streamwise $(x)$ direction and the spanwise $(y)$ direction. For the streamwise direction, a constant pressure-gradient is imposed to drive the fluid flow, whereas a zero pressure-gradient is for the spanwise direction.
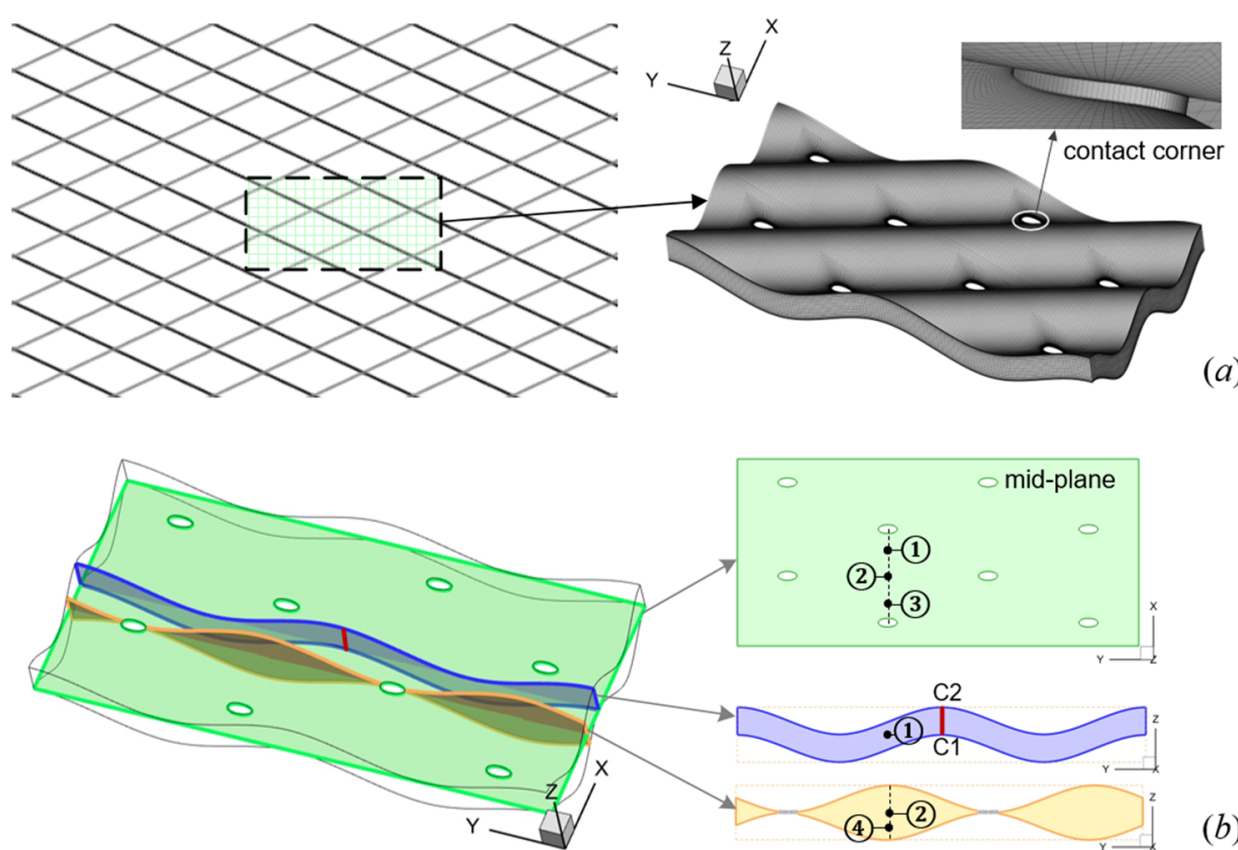

(b)
FIG. 2. (a) The extraction of a repetitive computational domain from the crosscorrugated channel and the hexahedral grids for the domain. (b) The deployment of numerical probes in the domain; $P$ - (1), $\mathrm{P}$-(2), and P-(3) are uniformly distributed between the two contact corners in the midplane, whereas P-(4) is placed at the downside of P-(2) with a distance of $b$. The velocity profile along the $\mathrm{C} 1$-to- $\mathrm{C} 2$ line will be sampled for the grid test. 

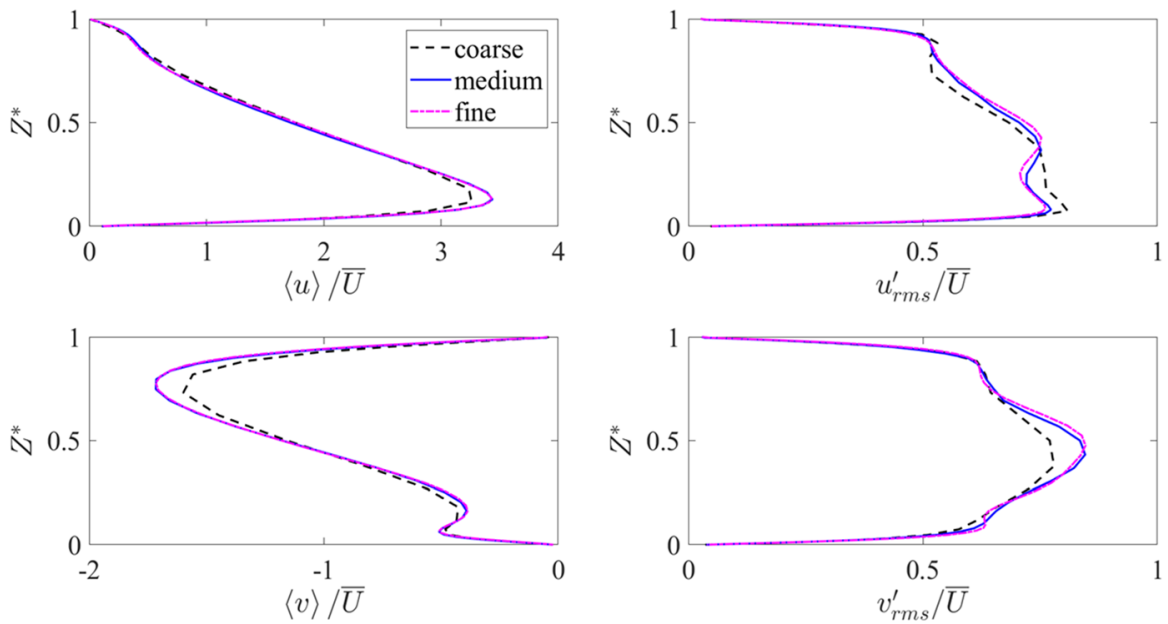

FIG. 3. A comparison of the grid resolution of different grid sizes at $R e=343.1$. The left side presents the time-averaged velocity components $\langle u\rangle,\langle v\rangle$, and $\langle w\rangle$, which are sampled from the C1-to-C2 line marked in Fig. 2(b); $Z^{*}=\left(z-z_{C 1}\right) /$ $\left(z_{C 2}-z_{C 1}\right)$. The root-mean-square of fluctuation components $u^{\prime}, v^{\prime}$, and $w^{\prime}$ is displayed on the right side. Velocities are normalized by the characteristic velocity $\bar{U}$.
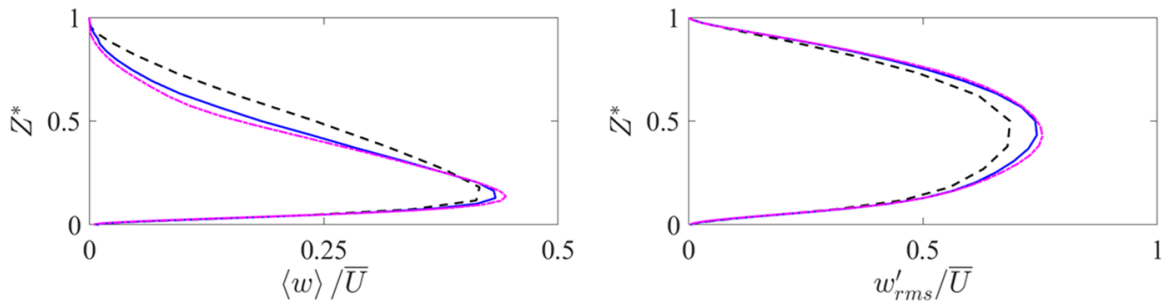

\section{Model validation}

Focke et l. $^{21}$ experimentally measured the hydraulic loss of fully developed flow in cross-corrugated channels over a wide range of Re, providing a suitable reference for the validation of our numerical results. The nondimensional Darcy friction factor is used to quantify the hydraulic loss of the channel, which is defined as follows:

$$
f=\frac{2 \Delta P_{L} d_{h}}{\rho \bar{U}^{2}}
$$

where $\Delta P_{L}$ is the pressure gradient per unit length.

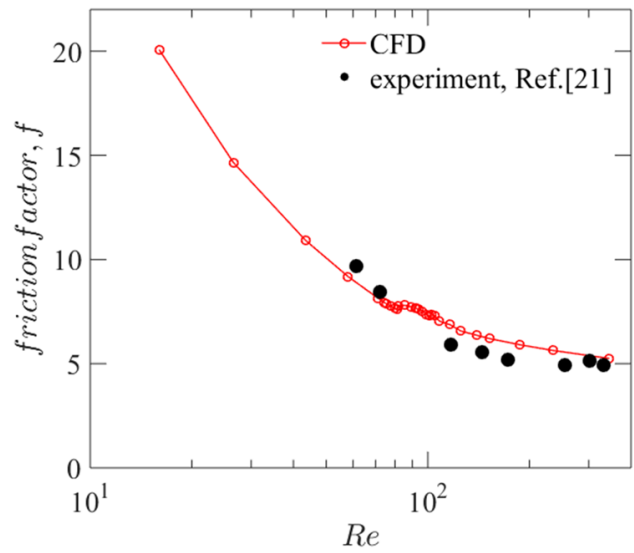

FIG. 4. A comparison of the friction factor between numerical and experimental results.
The model is validated by comparing the predicted friction factor with the experimental measurement presented by Focke et al., ${ }^{21}$ as shown in Fig. 4. Note that the inclination angle corresponding to the experimental results is $\beta=60^{\circ}$, which differs slightly from the value used in our simulations $\left(\beta=65^{\circ}\right)$. The results are in good agreement, proving the validity of our CFD results. Particularly, the CFD results suggest an abrupt increase of the friction factor at $R e$ around 77 , indicating the onset of flow unsteadiness.

\section{FLOW TRANSITIONS}

In this study, the power spectra density (PSD) and the phase space of pointwise time history of the flow velocity component are analyzed to reveal the nonlinearity of the Eulerian flow field. The power spectra are calculated by using the Fourier transform algorithm. The phase space of a pointwise velocity signal is plotted based on the time-delayed reconstruction technique.

\section{A. Steady mode $(R e<77.3)$}

Starting with $R e=9.3$, the flow remains steady until $R e \approx 77.2$. The time evolution of the velocity at P-(2) for $R e=75.6$ is shown in Fig. 5(a). The velocity is overshot at the beginning, and subsequently, its amplitude $|A|$ damps rapidly toward zero. The initial overshooting velocity field can be assumed as a temporal perturbation superposed to the base flow. According to the linear stability theory, the perturbation is amplified if the instability is critical; otherwise, the perturbation decays to zero. Apparently, Fig. 5(a) reflects the decay of the initial perturbation, which indicates that the flow instability is subcritical at $R e=75.6$. It is found that the decay of the velocity amplitude $|A|$ obeys the Landau equation, ${ }^{2}$ 


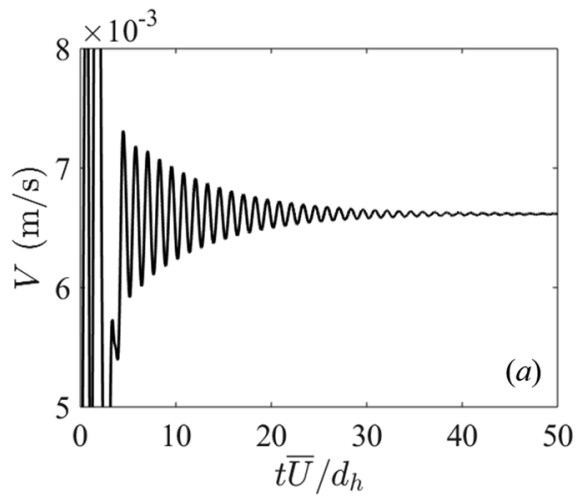

$$
\frac{d|A|}{d t}=\sigma|A|-g|A|^{3}
$$

where $\sigma$ is the growth rate of the fluctuation amplitude, and $|A|$ is the amplitude of velocity $U^{\prime}$. By calculating the $\sigma$ for a range of subcritical $R e$, we obtain a $\sigma$ vs Re diagram as shown in Fig. 5(b). The linear extrapolation of the curve to $\sigma=0$ yields a critical $R e$ of 81.6 , where a transition is expected to occur.

Figure 6 briefly describes the evolution of steady flow fields from $R e=9.3$ to $R e=75.6$. For $R e=9.3$, the driven pressure gradient is too small to cause any flow separation or recirculation. The streamline is fairly straightforward; see Fig. 6(a). As Re is increased

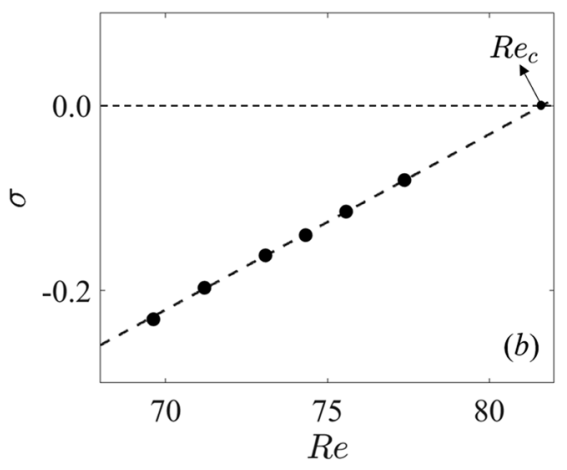

FIG. 5. (a) Time evolution of velocity $U$ at P-(2) $(R e=75.6)$. (b) The linear growth of $\sigma$ in the subcritical regime. The linear extrapolation to $\sigma=0$ yields a critical $R e_{c}$ $=81.6$.

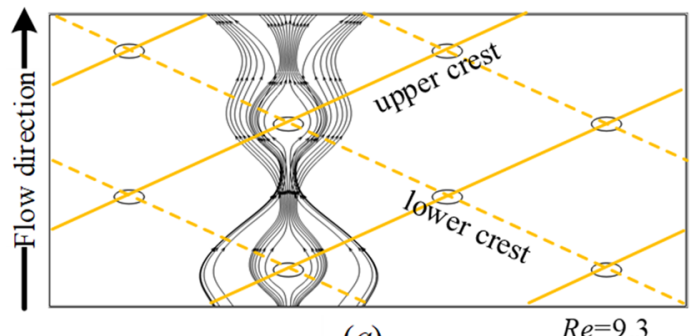

(a)

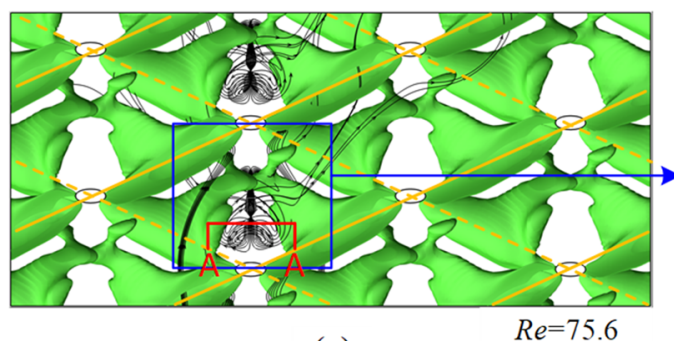

(c)

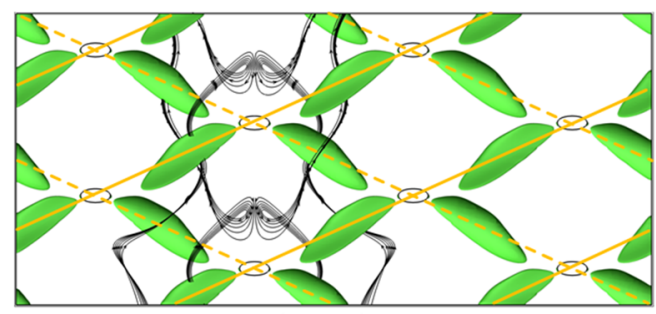

(b)

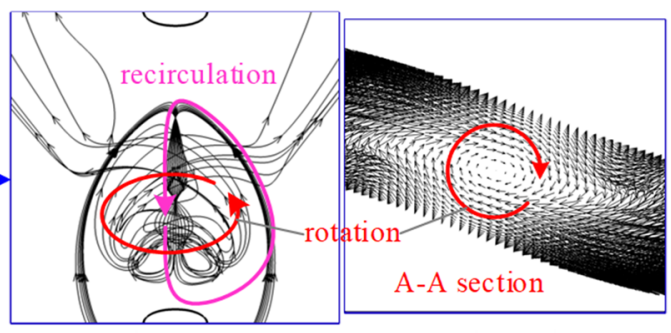

(d) (e)

FIG. 6. Top-view of the steady flow structures from $R e=9.3$ to $R e=75.6$. Note that orange lines mark the crests of upper and lower corrugated plates. Streamlines are colored in black. The vortexes and the shear layers are identified by the $Q$-criterion, defined as $Q=0.5(\|\Omega\|)^{2}-(\|S\|)^{2}$, where $\Omega$ and $S$ are the vorticity tensor and the rate-of-strain tensor, respectively. The isosurface of $Q=400$ (colored in green) is depicted to indicate the vorticity concentrated regions (stretched wall shears and free shears). (a) $R e=9.3$; low speed flow without flow separation. (b) $R e=26.5$; a pair of recirculation bubbles appears at the wake of contact corner; shear layers over the crest of the corrugated plate are stretched. (c) $R e=75.6$; separation bubbles are twisted together, forming the recirculating-and-rotating wake structure, enfolded by a pair of free shear layers. (d) A close-up of the wake structure of $R e=75.6$, which combines a motion of streamwise recirculation and spanwise rotation. (e) A close-up of the tangent velocity vectors in a spanwise cross section of the wake zone, which distinctively depict the spanwise rotation. 


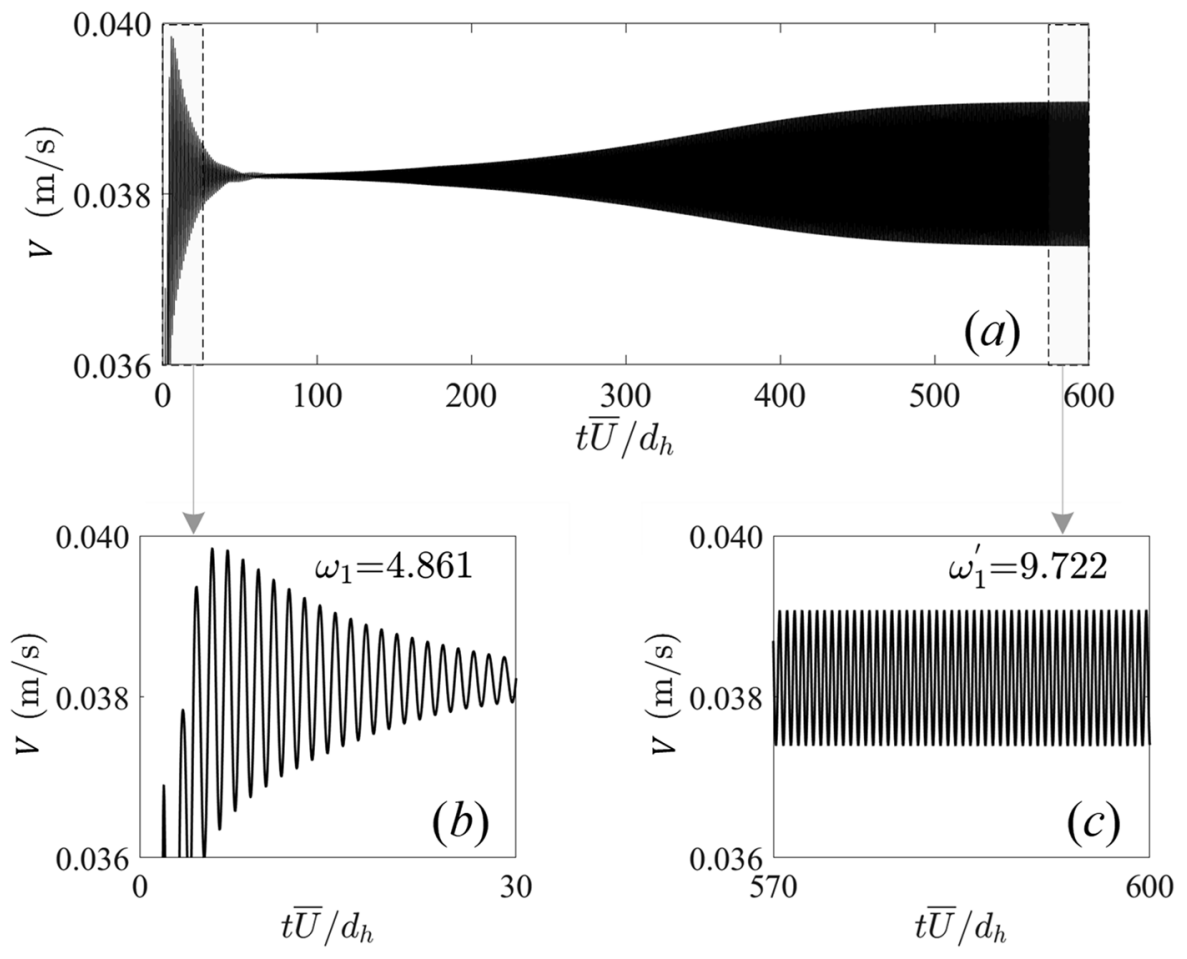

FIG. 7. Time history of the velocity at P-(2) $(R e=77.4)$. (a) is the full time history of the velocity. The beginning $\left(0<t \bar{U} / d_{h}<30\right)$ and the ending $(570$ $\left.<t \bar{U} / d_{h}<600\right)$ of the time history are clipped in (b) and (c), respectively. The frequency $\omega=1 / t$. gradient established behind the contact corner, while the spanwise rotation is caused by the opposite shear forces from the bounded free shear layers.

\section{B. Periodic- 1 mode $(77.3<R e<81.5)$}

The flow unsteadiness was detected when the Re goes beyond a critical value around 77.4. The time history of the velocity at P-(2) for $R e=77.4$ is shown in Fig. 7. At the beginning, the velocity fluctuation with a frequency of $\omega_{1}$ decays rapidly. Then, it is followed

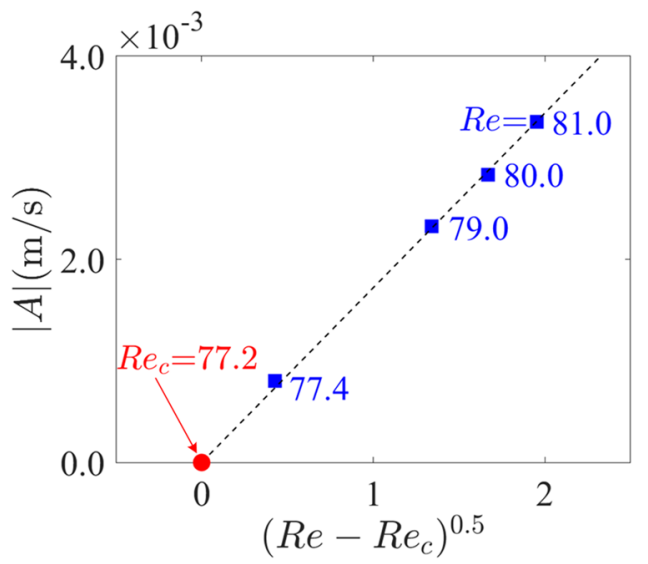

FIG. 8. The linear relationship between the fluctuation amplitude $|A|$ of the velocity at $\mathrm{P}$-(2) and $\left(R e-R e_{c}\right)^{0.5}$ under periodic-1 mode. The linear extrapolation to $|A|$ $=0$ yields a critical $R e_{c}=77.2$. by the amplification of a new frequency, $\omega_{1}^{\prime}$, and notably, $\omega_{1}^{\prime}=2 \omega_{1}$. The frequency $\omega_{1}^{\prime}$ is amplified over time to a constant amplitude. This indicates a Hopf bifurcation taking place at a critical $R e_{c}$ somewhere around 77.4. This critical $\mathrm{Re}_{c}$ is predictable, based on the Landau model. When the $R e$ is slightly over the $R e_{c}$, and the flow becomes unsteady, the fluctuation amplitude $|A|$ of the flow field can be approximated by $|A| \approx \varsigma\left(R e-R e_{c}\right)^{1 / 2}$, where $\varsigma$ is a constant. ${ }^{24}$

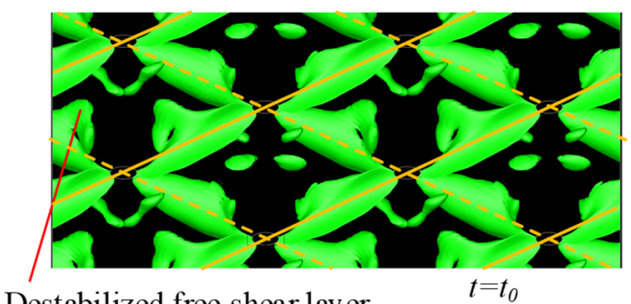

Destabilized free shear lay er

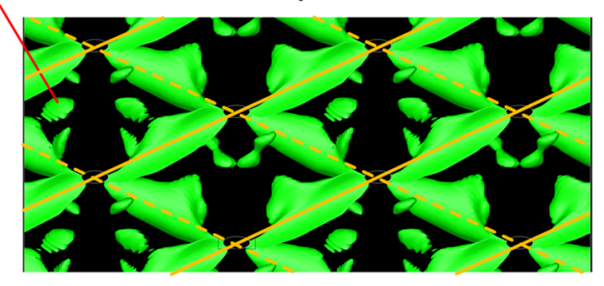

$t=t_{0}+0.2 T$

FIG. 9. Snapshots of the shear layer pattern $(R e=77.4)$ at two instant moments with a time difference of $0.2 T, T=1 / \omega_{1}^{\prime}$. Isocontour of $Q=1000$ (green) is used to indicate the shear layer. 


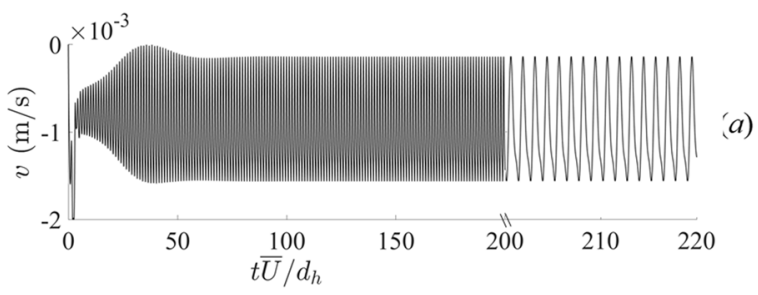

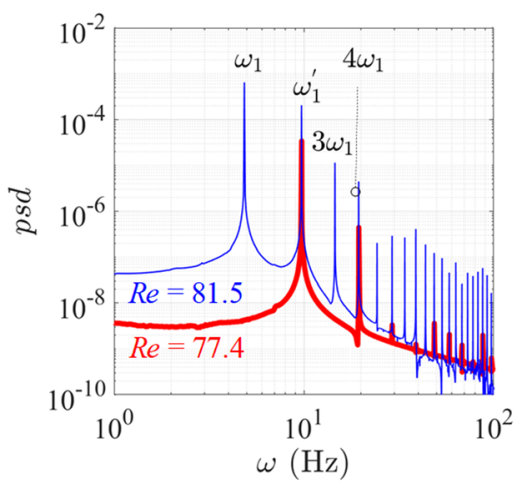

(b)

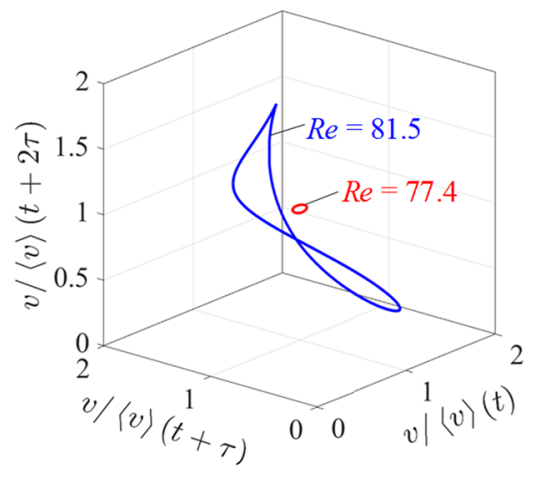

(c)
FIG. 10. (a) The time history of the velocity ( $v$-component) at P-(1) $(R e=81.5)$. (b) The power spectra density (PSD) for both $R e=81.5$ and $R e=77.4$. (c) The reconstructed phase space, the axes of which are normalized by its timeaveraged value $\langle v\rangle$.
Making use of this correlation, a diagram of $|A|$ vs $\left(R e-R e_{c}\right)^{1 / 2}$ is plotted in Fig. 8. The linear extrapolation to $|A|=0$ gives the critical $R e$ of 77.2, where a Hopf bifurcation occurs and the flow becomes weakly unstable. This unstable state is entitled as "periodic-1 mode."

In Fig. 9, the perturbation of free shear at $R e=77.4$ is displayed. The free shear layers are destabilized by the Kelvin-Helmholtz (K-H) instability, oscillating periodically with a frequency exactly equaling $\omega_{1}^{\prime}$. Therefore, the first transition corresponds to the periodical oscillation of the free shear layer.

\section{Periodic-2 mode $(81.5<R e<89.5)$}

The linear stability analysis in Fig. 5(b) suggests that it is possible for a transition to occur at the critical $R e_{c}=81.6$. This is verified by Fig. 10. When the Re gets close to 81.6, the flow dynamics dramatically differs from periodic- 1 mode, and a new periodic mode is established, which is then referred to as periodic- 2 mode. Figure 10 plots the time history of the velocity component acquired at $R e=81.5$, as well as the corresponding power spectra and phase space representation. For comparison purposes, the information for $R e=77.4$ is also conveyed in the figure. Figure 10(b) highlights a period doubling event occurring via this transition. At $R e=81.5$, a new frequency $\omega_{1}$ is produced, which is exactly a half of the dominant frequency $\omega_{1}^{\prime}$ for $R e=77.4$ (periodic-1 mode). The flow bifurcation is also evidenced in Fig. 10(c). For periodic-1 mode, the flow state develops into a limited circle with a very small amplitude. A new limited circle is established when $R e=81.5$ (periodic-2 mode), the period of which doubles that of periodic-1 mode. Hence,
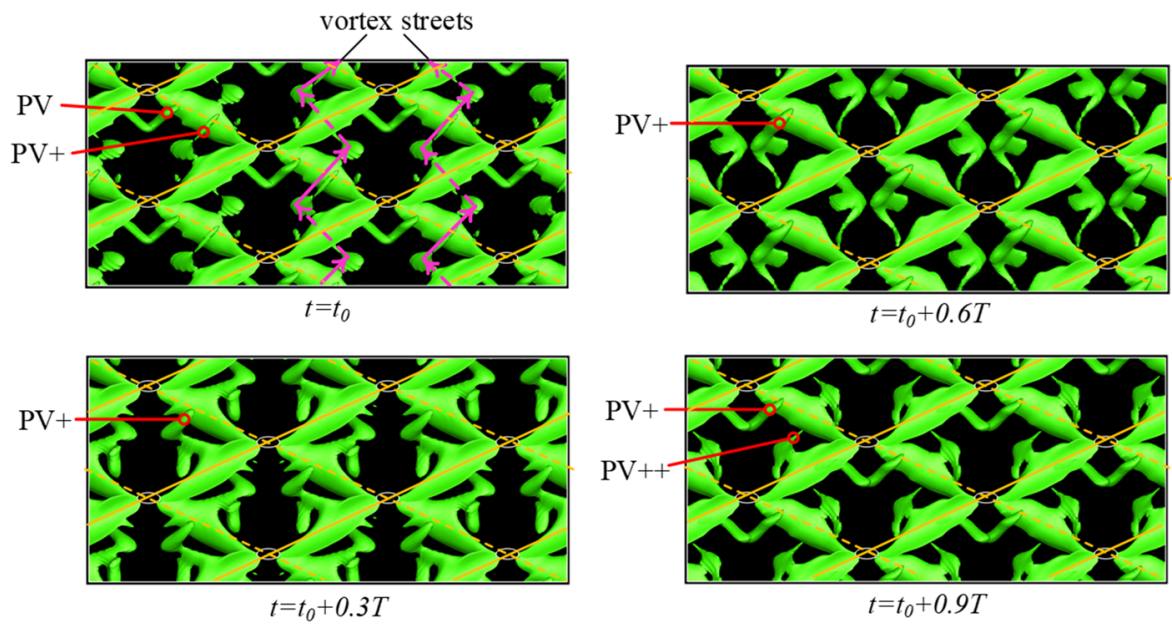

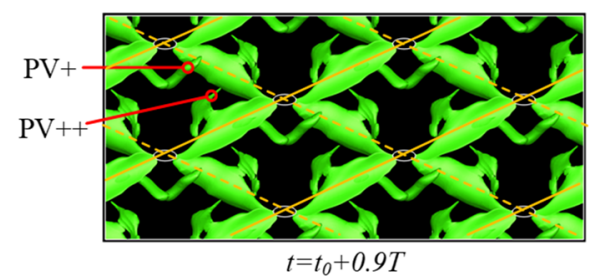

FIG. 11. Evolution of flow patterns $(Q$ $=1500$ ) for $R e=81.5$ (periodic-2) within a period of $T=\omega_{1}^{-1}$. "PV" denotes the primary vortex structure, and " + " represents the next generation of the "PV." The zig-zag vortex streets are marked by the pink lines and the solid line denotes the PV in the upper furrow, while the dashed line indicates that the PV is in the lower furrow. 

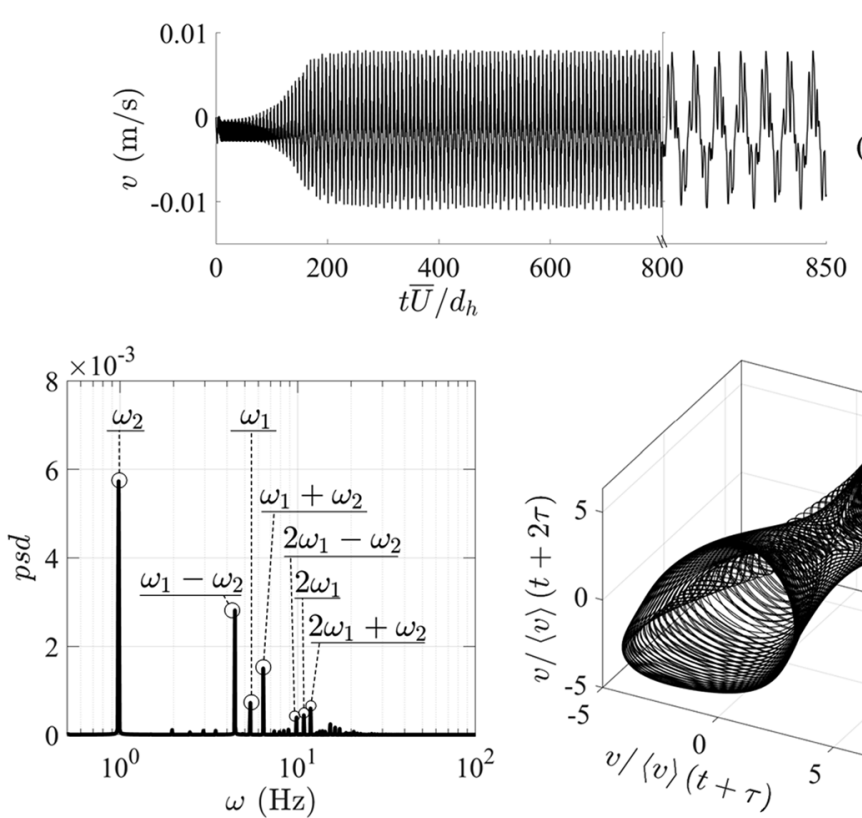

(b)

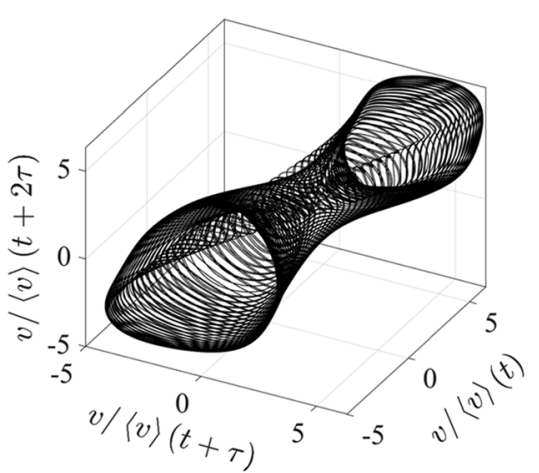

(c)
FIG. 12. (a) Time evolution of quasiperiodic $v$-component at P-(1) $(R e=94.0)$. (b) The corresponding power spectra density, which consists of two incommensurate frequencies of $\omega_{1}$ and $\omega_{2}$, as well as their linear combinations. (c) The corresponding phase space representation. it can be confirmed that a transition takes place at a critical $R e$ around 81.5 .

Figure 11 shows a time sequence of flow patterns at $R e=81.5$ within one period $\left(\Gamma=\omega_{1}^{-1}\right)$. The most striking feature is the type of the primary vortex ("PV" marked in Fig. 11) being produced. The vortexes orientate along the furrow subchannels, which appears to be similar to the "ox-horn" vortexes observed by Focke and Knibbe. ${ }^{5}$ The frequency of the primary vortex exactly equals $\omega_{1}$, corresponding to the primary frequency of periodic- 2 mode. The dynamic of the primary vortex in Fig. 11 is described as follows: At an instant $t=t_{0}$, a primary vortex (PV) is moving downstream. It is followed by the next generation of a primary vortex $(\mathrm{PV}+)$. The $\mathrm{PV}+$ is developing at $t=t_{0}+0.3 \Gamma$, while the $\mathrm{PV}$ is dissipated as it impinges on the walls in the upstream of the contact corner. Subsequently, the PV+ is shedding away from the free shear at $t=t_{0}+0.6 \Gamma$. At $t=t_{0}+0.9 \Gamma$, a periodic cycle is about to complete, and so the $\mathrm{PV}+$ almost reaches the same position of the PV at $t=t_{0}$. Meanwhile, the next generation of PV++ is conceived. When one period is completed at $t=t_{0}+\Gamma$ (not shown here), the flow structure completely recovers the topology shown at $t=t_{0}$. These primary vortexes sweep toward downstream, and their trajectory forms a zig-zag vortex street as indicated in Fig. 11. The primary vortex is initiated from the curved free shear layer, which should be the result of the centrifugal instability associated with the curved streamline in the free shear layer.
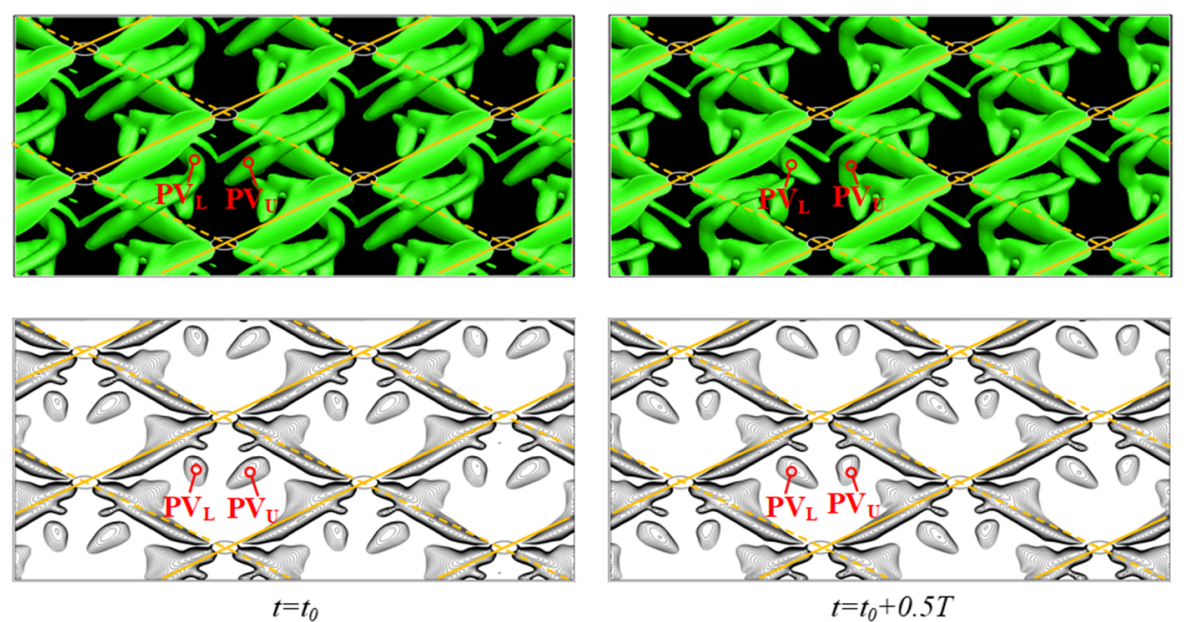

FIG. 13. The green isocontour $(Q$ $=2000$ ) shows instantaneous vortex patterns $(R e=94.0)$ in a time sequence with $T=1 / \omega_{2} . P V_{U}$ and $P V_{L}$ denote the primary vortexes in the upper furrow and the lower furrow, respectively. The lower figures show the $Q$ contour on the midplane of the channel, which clearly indicates the competition of the neighboring primary vortexes in a quasiperiodic cycle. 


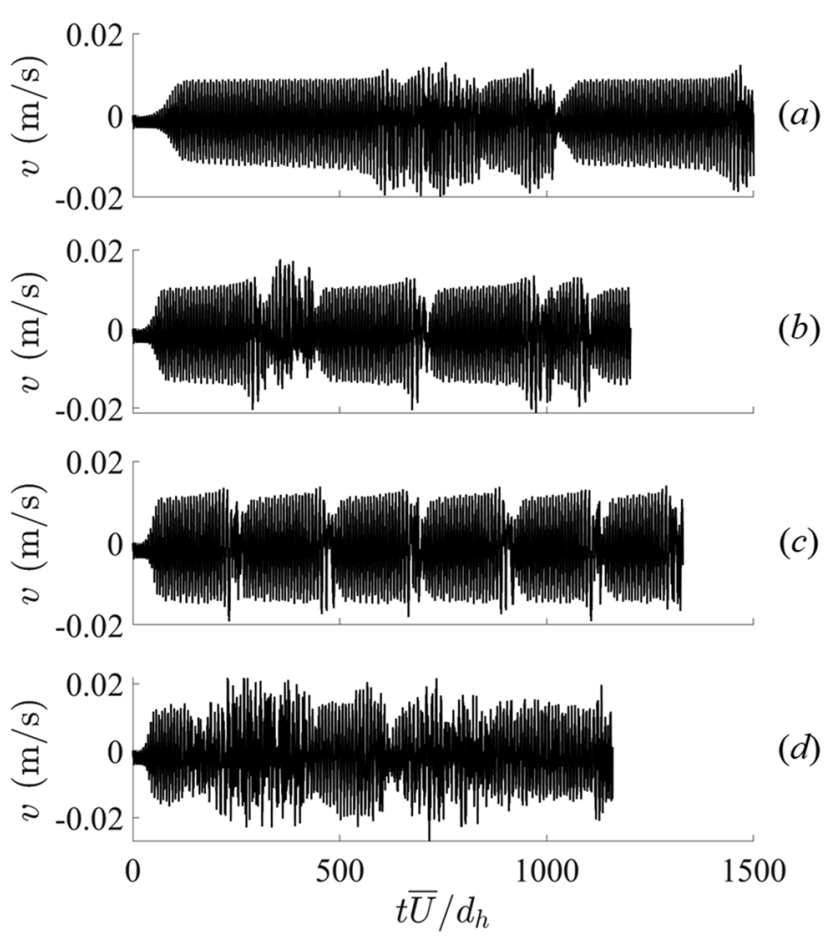

FIG. 14. Time histories of the $v$-component at $P$ - (1) for different $R e$. (a) $R e=96.5$, (b) $R e=100.8$, (c) $R e=102.7$, and (d) $R e=107.4$.

\section{Quasiperiodic mode $(91.7<R e<110.4)$}

The flow undergoes a third transition at a critical Re somewhere in between $89.5<R e_{c}<91.7$, developing into a quasiperiodic mode.

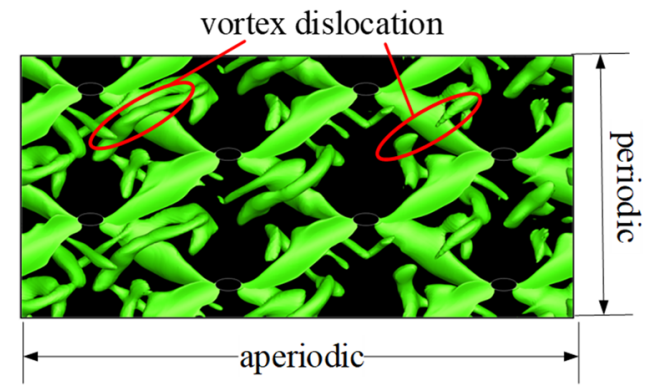

FIG. 15. Snapshot of the flow pattern $(Q=3000)$ for $R e=100.8$ at an instant of $t\langle U\rangle / d_{h}=412.5$ when the flow is experiencing the temporal intermittency, which indicates the temporal dislocation of the primary vortex structure in the spanwise direction.

The dynamics of the quasiperiodic mode is described in Fig. 12. The power spectra for $R e=94.0$ consist of two incommensurate frequencies, $\omega_{1}$ and $\omega_{2}$, as well as their linear combinations. In particular, the amplitude of the newly added frequency $\omega_{2}$ is highly pronounced. The phase space for the quasiperiodic mode, as shown in Fig. 12(c), is developed into a complex torus, which differs significantly from the limited circles shown in Fig. 10(c).

The main feature of the fluid dynamics in quasiperiod mode is represented by Fig. 13. It can be seen that the PV in the upper furrow $\left(\mathrm{PV}_{\mathrm{U}}\right)$ is not the same size as the $\mathrm{PV}$ in the lower furrow $\left(\mathrm{PV}_{\mathrm{L}}\right)$. At an instant $t=t_{0}, \mathrm{PV}_{\mathrm{U}}$ is larger than $\mathrm{PV}_{\mathrm{L}}$, while the opposite holds when $t=t_{0}+0.5 T$. It behaves like there is competition between the neighboring vortex streets. The competition frequency corresponds to the newly added frequency $\omega_{2}$ shown in Fig. 12(b).

Further increasing the $R e \geq 96.5$, it is found that the temporal intermittency emerges in the time history of the velocity, as

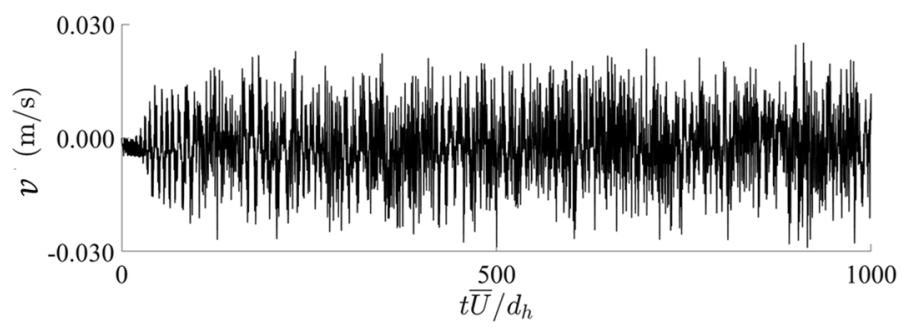

(a)

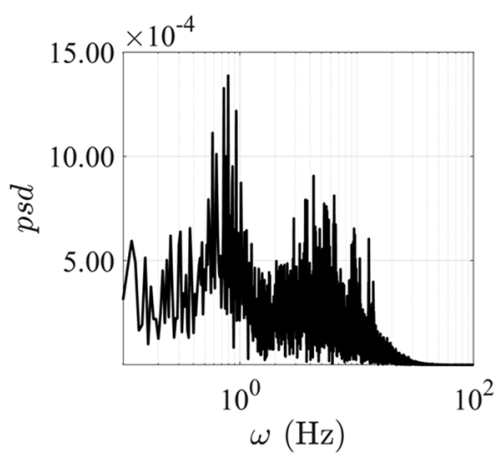

(b)

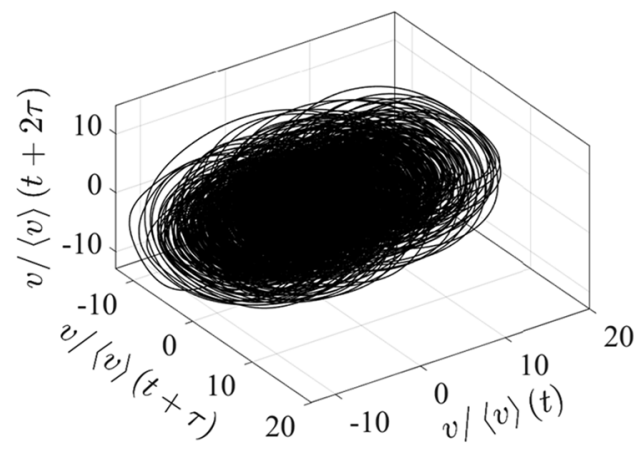

(c)
FIG. 16. (a) Time history of the $v$ component at $\mathrm{P}-(1)(R e=113.1)$. (b) The corresponding velocity spectra density. (c) The reconstructed phase space. 


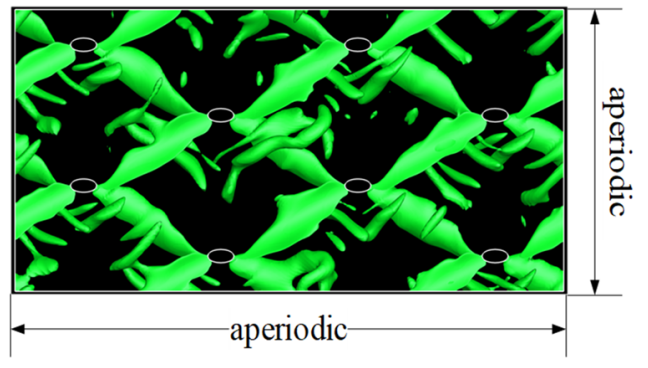

FIG. 17. Snapshot of the chaotic flow pattern $(Q=5000)$ at $R e=113.1$.

shown in Fig. 14. The occurrence of the intermittency appears to be more and more frequent as $R e$ is increased. Such a phenomenon is similar to the intermittency observed in the experiments of the Rayleigh-Bénard convection. ${ }^{25,26}$ Bergé et al. ${ }^{25}$ define the intermittency as temporal chaos that sets in continuously and manifests itself under the form of (apparently) random bursts interrupting quite regular laminar phases. This definition seems to be appropriate to describe the intermittent feature shown in Fig. 14. Gollub and Ben$\operatorname{son}^{26}$ also found that there are certain conditions under which the intermittency bursts the quasiperiodic orbits, leading the flow to chaos.

The flow pattern at an instant moment of the intermittent window is shown in Fig. 15. It can be seen that the spanwise flow loses periodicity at this moment, while the streamwise periodicity still persists. The vortex structure is dislocated along the spanwise direction, which leads to the temporal intermittency.

\section{E. Chaotic mode $(R e>113.1)$}

The time history of the velocity for $R e=113.1$ is presented in Fig. 16(a). The power spectra density, as shown in Fig. 16(b), is dominated by more than three incommensurate frequencies and broadband noises. In view of the phase space in Fig. 16(c), the space orbit is completely disordered, which is often referred to as a strange attractor.

A snapshot of the instantaneous vortex pattern for $R e=113.1$ is shown in Fig. 17. Apparently, the flow has lost periodicity both streamwise and spanwise. The primary vortex structure can still be observed. However, unlike the behavior of the quasiperiodic mode, the vortex streets for $R e=113.1$ fail to correlate from one to another, and hence, the competition between vortex streets becomes disordered. For this case, the chaos seems to be caused by the disordered interaction of the vortex streets, rather than the onset of new instability or the formation of any new structures. So from this point of view, the onset of chaos at such a low Re condition, as WilliamsStuber and Gharib ${ }^{27}$ concluded, is most likely to result from the competitions of local orders.

Of great interest is to see how the chaos further develops as Re is increased. By increasing Re to 343.1, the chaotic feature becomes more significant, as illustrated by Fig. 18(a). The flow is now characterized by dense vortex structures with different scales. In particular, it is observed that furrow-wise vortex arrays are formed over the crests of the corrugated plate. A cut section of the furrow is shown in Fig. 18(b), from where it is seen that these vortexes are counterrotating, indicating that the formed vortex arrays are most likely to be of the Taylor-Görtler type. In addition, it is observed that necklace vortexes are frequently formed in the upstream of the contact corner, as illustrated in Fig. 18(c). The necklace vortex structure often appears around a wall junction. ${ }^{28}$ Due to the locally adverse pressure gradient, the necklace vortex originating in the separation region stretch around the contact corner and fold around the upstream part of it.

\section{ROUTE TO CHAOS}

This section is deemed to summarize the flow transition process in a cross-corrugated channel. A spectra map, a series of Poincaré sections, and a bifurcation diagram are developed to illustrate the route of transition from steady to chaos with the increase of the control parameter Re.

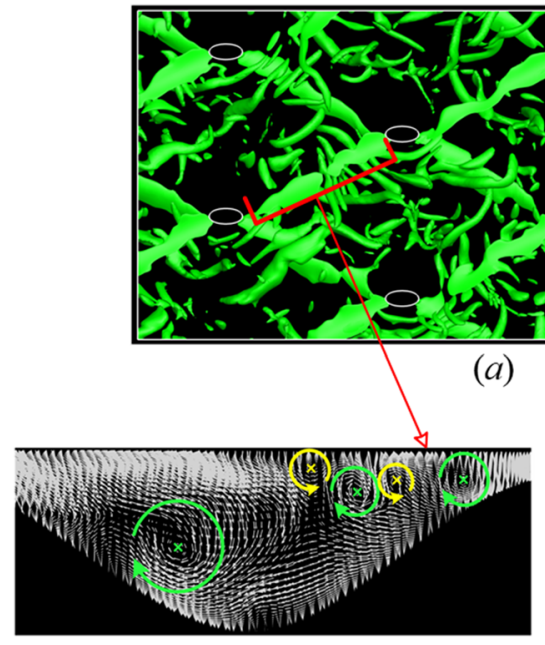

(b)
FIG. 18. (a) Snapshot of the vortex structure $\left(Q=2 \times 10^{5}\right)$ for $R e=343.1$. (b) The velocity vector for a cross section of the furrow, which shows the counter-rotating feature of the furrow-wise vortex arrays. (c) The necklace vortex is formed in the upstream of the contact corner. 


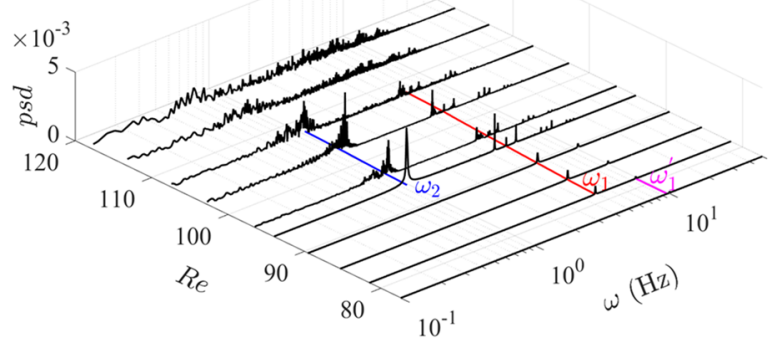

(a)

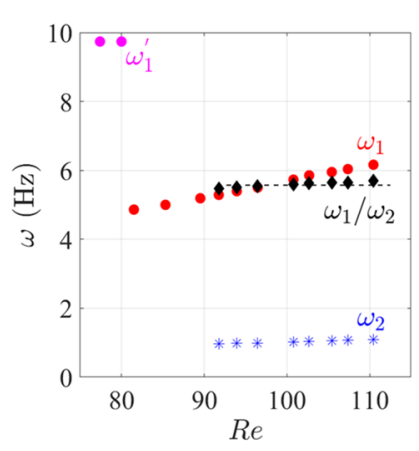

(b)
FIG. 19. (a) The variation of the velocity spectra ( $v$-component at $P$ - $(1)$ ) along with the increase of $R e$. (b) The incommensurate frequencies of $\omega_{1}$ and $\omega_{2}$, as well as their ratio $\omega_{1} / \omega_{2}$, which is locked to a constant value around 39/7.

\section{A. Spectra map}

In Fig. 19(a), the power spectra density of the velocity for different $R e$ are collected, providing an overview of the development of flow nonlinearity. For periodic-1 mode, only one fundamental frequency $\omega_{1}^{\prime}$ exists, and it is replaced by a new dominant frequency $\omega_{1}\left(\omega_{1}=2 \omega_{1}^{\prime}\right)$ when the flow switches to periodic-2 mode. The quasiperiodic mode takes place when an incommensurate frequency $\omega_{2}$ is added to the spectra. The intermittency brings noise to the spectra. The noise grows with $R e$ due to the frequent occurrence of intermittencies. At the onset of chaos, the spectra are characterized by more than three incommensurate frequencies and broadband noises. The path to chaos discussed above admits the Ruelle-Takens-Newhouse scenario. ${ }^{29}$ Figure 19(b) plots the incommensurate frequencies from low $R e$ to high $R e$. It is interesting to see that both $\omega_{1}$ and $\omega_{2}$ are almost proportional to $R e$. The values of the ratio $\omega_{1} / \omega_{2}$ are also plotted in the figure and are locked to a constant value. This phenomenon is often termed "phase-locking." Since the two frequencies are incommensurate, the ratio $\omega_{1} / \omega_{2}$ is an irrational value, which is estimated to be around $39 / 7$.

\section{B. Poincaré section}

This section displays the intersecting points between the phase space orbit and a hyperplane, which gives a simple but clear manifestation of the dynamics inscribed on the phase space torus. Taking the phase space in Fig. 10 (c) as an example, to get its Poincaré sections, the hyperplane is chosen as the $v /\langle v\rangle(t)=1$ so that the intersection between the limited circle and the hyperplane leaves two points in the section, as shown in Fig. 20(a). Likewise, the Poincaré sections for other modes are plotted in Fig. 20, which altogether depict the route to chaos of the cross-corrugated channel flow. For periodic modes $(R e=77.4$ and $R e=81.5)$, the limited circles leave two single points in the section. For the quasiperiodic mode at $R e=94.0$, a complex but ordered torus is formed, which is the base torus of the quasiperiodic mode. At $R e=96.5$, the torus is interrupted by the temporal intermittency. The state departs from the base torus once the intermittency sets in, while it is retrieved back to the base torus at the end of an intermittency window. In the section of $R e=113.1$, no regular torus can be found, indicating that the flow is chaotic.

\section{Bifurcation diagram}

A bifurcation diagram is depicted in Fig. 21 by plotting the peaks and valleys of the velocity history against the control parameter $R e$. The bifurcations can be clearly viewed at each critical $R e$. At $R e_{c 1}$, a bifurcation occurs due to the excitation of the K-H mode. The second bifurcation takes place at $R e_{c 2}$, corresponding to the formation of primary furrow-wise vortexes. The third bifurcation at $R e_{c 3}$ leads the flow into quasiperiodic mode, characterized by the competition between adjacent vortex streets. The peaks and valleys fall into
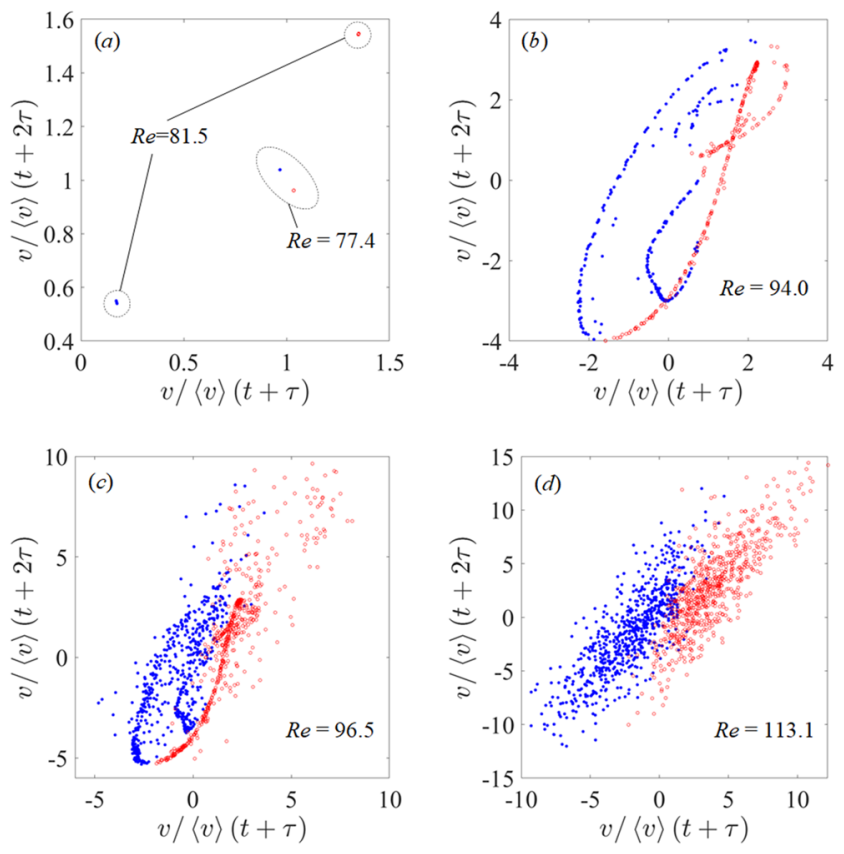

FIG. 20. Poincaré sections for different $R e$. The blue circle and red circle represent the intersection points when the phase space orbit ( $v$-component at P-(1)) inward and outward intersects with the hyperplane, respectively. (a) Periodic-1 mode $(R e=77.4)$ and periodic-2 mode $(R e=81.5)$. (b) Base quasiperiodic mode ( $R e$ $=94.0)$. (c) Quasiperiodic mode with intermittency $(R e=96.5)$. (d) Chaotic mode $(\operatorname{Re}=113.1)$. 


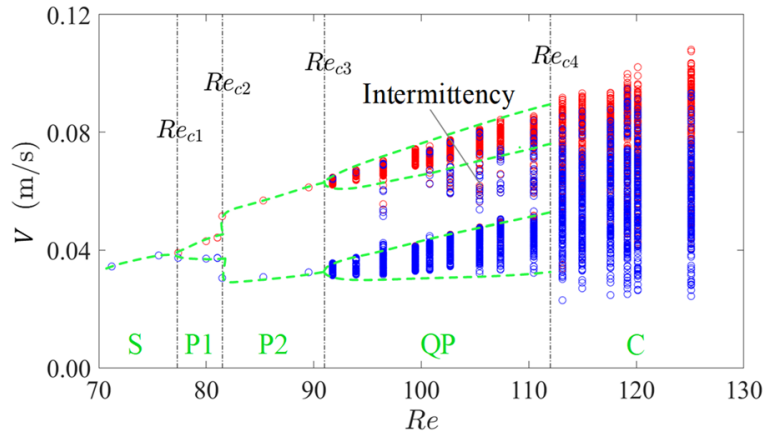

FIG. 21. Bifurcation diagram drafted by plotting the max-min of the velocity history (at P-(4)) against the control parameter Re. Red dots and blue dots represent the max and the min of the velocity history, respectively. The diagram is divided into five regions by critical Reynolds numbers, including $S$ (steady mode), $P 1$ (periodic1 mode), P2 (periodic-2 mode), QP (quasiperiodic mode), and C (chaotic mode).

a narrow band. A few points falling outside of the bands correspond to the temporal intermittencies. $R e_{c 4}$ is the critical point where the transition to chaos finally takes place. In chaotic mode, the red dots (peaks) and the blue dots (valleys) are overlapped, and no distinguishable band for peaks or valleys can be found because the velocity fluctuation is random and disorder.

\section{CONCLUSIONS}

The flow transition in a cross-corrugated channel with inclination angle $\beta=65^{\circ}$ was studied numerically in this paper. The study reveals how this channel flow develops from steady to chaos by increasing the control parameter $R e$. The entire transition sequence and associated fluid dynamics are described as follows:

The flow remains steady and laminar at subcritical $R e(R e$ $<77.3$ ). A three-dimensional wake structure is found behind each contact corner, manifesting itself in a recirculating-and-rotating pattern. The wake is enfolded by two pieces of curved free shear layers.

The first transition is a supercritical Hopf bifurcation, leading the flow into periodic- 1 mode. The linear stability analysis based on the Landau model suggests that the critical $R e_{c 1}$ corresponding to this bifurcation is 77.3. Physically, the Kelvin-Helmholtz instability is responsible for the first transition, which destabilizes the curved free shear layers.

The second transition takes place at $R e_{c 2} \sim 81.5$, leading the flow into another periodic mode (periodic-2 mode), the period of which doubles that of periodic- 1 mode. The linear stability analysis again gives a close prediction of critical $R e$. In view of the flow structure, furrow-wise vortexes are formed under periodic- 2 mode. These primary vortexes result from the centrifugal instability of the curved shear layer. The trajectory of these vortexes forms "zig-zag" streamwise vortex streets.

The transition from periodic- 2 to quasiperiodic occurs at $R e_{c 3}$ $\left(89.5<R_{c 3}<91.7\right)$. Two incommensurate frequencies and large numbers of their linear combinations appear in the power spectra. The ratio between the two incommensurate frequencies are locked to a constant value during the quasiperiodic mode. The competition between the adjacent vortex streets is observed. The competition has a frequency which equals to the newly added frequency. As $R e$ is further increased, the temporal intermittency arises and bursts the base quasiperiodic state. The intermittency is due to the local dislocation of the vortex street. The intermittency appears more and more frequent as $R e$ is increased.

The flow finally becomes chaos at $R e_{c 4}\left(110.4<R e_{c 4}<113.1\right)$. At the onset of chaos, the power spectra of the velocity are characterized by more than three incommensurate frequencies and broadband noise. The flow structure loses periodicity both in spanwise and in streamwise directions. Further increasing $R e$ to 343.1, the chaos becomes more strengthened. Taylor-Görtler like vortexes are observed over the crest of the corrugated plate. In addition, the necklace vortexes are frequently created in the upstream of the contact corner.

In summary, the sequence of transition for the considered channel flow is as follows: steady, periodic-1, periodic-2, quasiperiodic (phase-locking), quasiperiodic with intermittency, and finally chaos. The route admits the Ruelle-Takens-Newhouse scenario ${ }^{29}$ in that chaos is developed by incommensurate bifurcations. Moreover, the transition from quasiperiodic mode to chaotic mode is accompanied with a rising number of temporal intermittencies. The phenomenon is comparable to one of the transition routes found in the Rayleigh-Bénard convection. ${ }^{2}$

\section{ACKNOWLEDGMENTS}

The research presented in this paper was funded by the European Union's Horizon 2020 research and innovation programme under the Marie Skłodowska-Curie Grant Agreement No. 713683. The financial support is gratefully acknowledged.

\section{REFERENCES}

${ }^{1}$ R. K. Shah and W. W. Focke, "Plate heat exchangers and their design theory," Heat Transfer Equip. Des. 227, 254 (1988).

${ }^{2}$ L. Spiegel and M. Duss, "Structured packings," in Distillation (Elsevier, 2014), pp. 145-181.

${ }^{3}$ G. Xiao, T. Yang, H. Liu, D. Ni, M. L. Ferrari, M. Li, Z. Luo, K. Cen, and M. Ni, "Recuperators for micro gas turbines: A review," Appl. Energy 197, 83 (2017).

${ }^{4}$ A. Ghanem, T. Lemenand, D. Della Valle, and H. Peerhossaini, "Static mixers: Mechanisms, applications, and characterization methods-A review," Chem. Eng. Res. Des. 92, 205 (2014).

${ }^{5}$ W. W. Focke and P. G. Knibbe, "Flow visualization in parallel-plate ducts with corrugated walls," J. Fluid Mech. 165, 73 (1986).

${ }^{6}$ Y.-C. Tsai, F.-B. Liu, and P.-T. Shen, "Investigations of the pressure drop and flow distribution in a chevron-type plate heat exchanger," Int. Commun. Heat Mass Transfer 36, 574 (2009).

${ }^{7}$ S. Freund and S. Kabelac, "Investigation of local heat transfer coefficients in plate heat exchangers with temperature oscillation IR thermography and CFD," Int. J. Heat Mass Transfer 53, 3764 (2010).

${ }^{8}$ K. Sarraf, S. Launay, and L. Tadrist, "Complex 3D-flow analysis and corrugation angle effect in plate heat exchangers," Int. J. Therm. Sci. 94, 126 (2015).

${ }^{9}$ S. Chapaloglou, A. Nikolopoulos, N. Nikolopoulos, S. Karellas, and P. Vourliotis, "Numerical analysis of a GPHE's hydrodynamic and thermal characteristics, by applying an iterative procedure for the thermal boundary conditions," Int. J. Heat Mass Transfer 118, 88 (2018).

${ }^{10}$ H. Blomerius, C. Hölsken, and N. K. Mitra, "Numerical investigation of flow field and heat transfer in cross-corrugated ducts," J. Heat Transfer 121, 314 (1999).

${ }^{11}$ J. Lee and K.-S. Lee, "Flow characteristics and thermal performance in chevron type plate heat exchangers," Int. J. Heat Mass Transfer 78, 699 (2014). 
${ }^{12}$ N. K. Ghaddar, K. Z. Korczak, B. B. Mikic, and A. T. Patera, "Numerical investigation of incompressible flow in grooved channels. Part 1. Stability and self-sustained oscillations," J. Fluid Mech. 163, 99 (1986).

${ }^{13}$ E. P. L. Roberts, "A numerical and experimental study of transition processes in an obstructed channel flow," J. Fluid Mech. 260, 185 (1994).

${ }^{14}$ L. Kaiktsis, G. E. Karniadakis, and S. A. Orszag, "Onset of three-dimensionality, equilibria, and early transition in flow over a backward-facing step," J. Fluid Mech. 231, 501 (1991).

${ }^{15}$ G. E. Karniadakis, B. B. Mikic, and A. T. Patera, "Minimum-dissipation transport enhancement by flow destabilization: Reynolds' analogy revisited," J. Fluid Mech. 192, 365 (1988).

${ }^{16}$ A. M. Guzmán and C. H. Amon, "Transition to chaos in convergingdiverging channel flows: Ruelle-Takens-Newhouse scenario," Phys. Fluids 6, 1994 (1994).

${ }^{17}$ E. P. L. Roberts and M. R. Mackley, "The development of asymmetry and period doubling for oscillatory flow in baffled channels," J. Fluid Mech. 328, 19 (1996).

${ }^{18}$ Y. Sui, C. J. Teo, and P. S. Lee, "Direct numerical simulation of fluid flow and heat transfer in periodic wavy channels with rectangular cross-sect," Int. J. Heat Mass Transfer 55, 73 (2012).

${ }^{19}$ F. Ducros, F. Nicoud, and T. Poinsot, "Wall-adapting local eddy-viscosity models for simulation in complex geometries," in 6th ICFD Conference on Numerical Methods for Fluid Dynamics, 1998.
${ }^{20}$ J. Fröhlich, C. P. Mellen, W. Rodi, L. Temmerman, and M. A. Leschziner, "Highly resolved large-eddy simulation of separated flow in a channel with streamwise periodic constrictions," J. Fluid Mech. 526, 19 (2005).

${ }^{21}$ W. W. Focke, J. Zachariades, and I. Olivier, "The effect of the corrugation inclination angle on the thermohydraulic performance of plate heat exchangers," Int. J. Heat Mass Transfer 28, 1469 (1985).

${ }^{22}$ N. H. Packard, J. P. Crutchfield, J. D. Farmer, and R. S. Shaw, "Geometry from a time series,” Phys. Rev. Lett. 45, 712 (1980).

${ }^{23}$ L. D. Landau, "On the problem of turbulence," Dokl. Akad. Nauk SSSR 44, 339 (1944).

${ }^{24}$ M. Provansal, C. Mathis, and L. Boyer, "Bénard-von Kármán instability: transient and forced regimes," J. Fluid Mech. 182, 1 (1987).

${ }^{25}$ P. Bergé, M. Dubois, P. Mannevillel, and Y. Pomeau, "Intermittency in Rayleigh-Bénard convection," J. Phys. Lett. 41, 341 (1980).

${ }^{26} \mathrm{~J}$. Gollub and S. V. Benson, "Many routes to turbulent convection," J. Fluid Mech. 100, 449 (1980).

${ }^{27} \mathrm{~K}$. Williams-Stuber and M. Gharib, "Transition from order to chaos in the wake of an airfoil," J. Fluid Mech. 213, 29 (1990).

${ }^{28}$ C. V. Seal and C. R. Smith, "Visualization of a mechanism for three-dimensional interaction and near-wall eruption," J. Fluid Mech. 394, 193 (1999).

${ }^{29}$ D. Ruelle and F. Takens, "On the nature of turbulence," Commun. Math. Phys. 20, 167 (1971). 